Factors influencing provision of pharmacist discharge prescription transcription services and authorisation requirements of pharmacist-written prescriptions

Sewell, GJ

http://hdl.handle.net/10026.1/3733

International Journal of Pharmacy Practice

All content in PEARL is protected by copyright law. Author manuscripts are made available in accordance with publisher policies. Please cite only the published version using the details provided on the item record or document. In the absence of an open licence (e.g. Creative Commons), permissions for further reuse of content should be sought from the publisher or author. 


\section{Factors influencing provision of pharmacist discharge prescription transcription services and authorisation requirements of pharmacist-written prescriptions}

\section{R. J. HoBson and G. J. Sewell \\ Introduction Recent reports describe the development of pharmacists tran- scribing discharge prescriptions, ${ }^{1,2}$ but there is no published information on factors influencing the decision whether or not to provide a service. \\ Anecdotal evidence suggests con- flicting opinion on the issue of whether a medical practitioner should be legal- ly required to co-sign a prescription written by a pharmacist. \\ A survey was distributed to one acute hospital from each UK NHS trust to examine these two issues.}

Method The questionnaire was developed after literature review and visits to hospitals running IDPTS. It contained open and closed questions. Face-to-face interviews with pharmacists were used for validation. Single hospitals in each UK NHS trust were identified using the UK arug information pharmacists' group cirectory and the Chemist of Druggist directory. Ambulance trusts, learning disabilities trusts and community hospitals were excluded.

The questionnaire was piloted in 20 randomly selected hospitals (July 2001), and then distributed (August 2001). It was addressed to the chief pharmacist, principal pharmacist or clinical services manager according to the name found.

Responses were coded and analysed using the statistical package for the social sciences (SPSS) version 10.

Results Of 206 pharmacists contacted, 135 (66 per cent) responded, of whom 49 (36 per cent) reported that they were offering PDPTS.

The most frequently cired reason for not implementing a transcription service was insufficient resources (18), followed by developing other services in preference to PDPTS (7). Other reasons given were no plans/discussion (6), lack of funding (5), electronic prescribing development will make PDPTS redundant, considered an administrative role and that it was for the doctor's benefit only.

The most common reason for implementing a transcribing service was to reduce delays in the discharge pro-

\section{FoCAL POINTS}

$\square$ A questionnaire survey was distributed to one acute hospital from each UK NHS trust to examine the factors influencing the decision whether to provide pharmacist discharge prescription transcription services (PDPTS)

$\square$ Sixty-six per cent of pharmacists responded and 36 per cent of these reported that they were offering PDPTS; the most frequently cited reason for not implementing a transcription service was insufficient resources, followed by developing other services in preference to PDPTS

$\square$ The most common reason for implementing a transcribing service was to reduce delays in the discharge process, followed by improved accuracy/decreased errors/improved quality

$\square$ The majority of HPDs ask the doctor to co-sign the pharmacist written prescriptions

$\square$ This survey has shown that there is no consensus on authorisation requirements; until legislation is changed to permit pharmacists to prescribe dependently, this issue will remain unresolved

cess (72.9 per cent, 35), followed by improved accuracy/decreased errors/ improved quality (50 per cent, 24). Other reasons included release of junior doctor time (33.3 per cent, 16), increased efficiency, cost savings from reuse of patient's own drugs, enhanced pharmacist's role, improved communications with primary care, decreased duplicate prescribing, save nursing time, enhanced counselling opportunities, risk management and improved patient care.

Most HPDs ask the doctor to cosign the pharmacist-written prescriptions (31, 64.6 per cent); however, 10 (20.8 per cent) did not and seven (14.6 per cent) said that they sometimes did (1=missing data). Those that did not ask the doctor to co-sign often asked the doctor to indicate in another manner that the prescription was required. Seven HPDs used verbal authorisation, four HPDs asked the doctor to sign the in-patient drug chart.

Discussion A main reason for not having PDPTS was lack of resources (18). The most common reasons found in this study supporting the provision of PDPTS have also been identified in published literature..$^{1,2}$

If the pharmacist is writing the dis- charge prescription, there is a risk management issue, as the pharmacist is no longer performing the clinical check role. Some hospitals have overcome this issue by swapping the prescribing and checking roles with the doctor.1,3 This survey has shown that there is no consensus on authorisation requirements. Anecdotal evidence from this survey suggests using the doctor as a second clinical check when they cosign the prescription was not successful. The value of signatures on in-patient drug charts has also been questioned. The in-patient drug chart is not a prescription, but the authority to administer a medicine. Cousins and Luscombe have suggested that as long as the authority to administer each drug can be traced back to either a written protocol or a general practitioner, there may be an absolute requirement to have a doctor countersign the items on the inpatient chart. 4 This theory could also be extended to the discharge prescription. Until legislation is changed to permit pharmacists to prescribe dependently, this issue will remain unresolved.

A limitation of this study is it would have been preferable to send the questionnaire to every UK hospital as opposed to one per trust.
Department of Pharmacy and Pharmacology, University of Bath, and Swindon and Marlborough NHS Trust

R. J. Hobson

Department of Pharmacy and Pharmacology, University of Bath, and Plymouth Hospitals NHS Trust

G. J. Sewell

Int J Pharm Pract 2002:10(suppl):R62

\section{References}

1. Boorman S, Cairns C. Another way forward for pharmaceutical care: a team-based clinical pharmacy service. Pharm J 2000;264: 343-6.

2. Cattell R, Conroy

C, Sheikh A.

Pharmacist integration into the discharge process: a qualitative and quantitative impact assessment. Int J Pharm Pract 2001;9: 59-64.

3. Milliken B, Rea Y.

A clinical

pharmacist's

contribution to discharge planning and patient counselling. Pharm J 1997;258:777-8. 4. Cousins DH, Luscombe DK. A new model for hospital pharmacy practice. Pharm J

1996;256:347-51. 


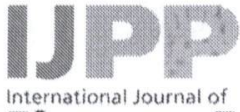

Pharmacy Practice

IJPP 2003; 11: 89-95

(C) 2003 Int J Pharm Pract

Received August 4, 2002

Accepted February 25,2003

DOI 10.1211/0022357021224

ISSN 0961-767

\section{A national survey of pharmacist transcribing of discharge prescriptions}

\author{
Rachel J. Hobson and Graham J. Sewell
}

\begin{abstract}
Objective To provide quantitative data on pharmacist discharge prescription transcription service (PDPTS) provision in UK hospitals.

Method Postal questionnaire survey of clinical pharmacy managers.

Setting Selection criteria included one hospital in each acute trust in the UK.

Key findings The response rate was $66 \%$ (135/206). In mid-2001, a PDPTS was provided by 49 hospital pharmacy departments (36\%). PDPTS was the most common prescribing activity undertaken by pharmacists, followed by a prescription amendment policy $(29 \%)$, prescribing in pre-admission clinics (18\%) and re-writing drug charts (15\%). 59 departments (44\%) did not undertake any prescribing activity.

Of the 86 non-transcribing hospitals, $69 \%$ undertook no prescribing activity (range $=0$ to 3 prescribing activities). Transcribing hospitals offered a wider range of prescribing activities (range $=1$ to 8 prescribing activities). A weak relationship was found between the number of pharmacists employed per hospital and the number of prescribing activities undertaken (correlation coefficient $=0.208, P=0.018)$. The most frequently used PDPTS model $(78 \%)$ involved pharmacists transcribing the discharge prescriptions for their own wards. The number of pharmacists transcribing discharge prescriptions per hospital ranged from 1 to 89 (mean=8, mode=2, median $=5,25 \%$ percentile $=2,75 \%$ percentile $=10$ ). The majority of pharmacists $(52 \%)$ reported writing less than five prescriptions per day; 35\% were writing 5-10 prescriptions per day. The most common training requirement for pharmacists to start transcribing was an in-house training programme $(55 \%)$. The majority of departments $(80 \%)$ did not re-assess the ability of their pharmacists to transcribe.

Conclusion Hospital pharmacy departments in the UK have started to take on prescribing roles, especially transcribing discharge prescriptions. However, it would appear that the majority of the PDPTS schemes are not being run extensively throughout the hospitals. It is of concern that the principles of clinical governance are not being met in terms of training and re-assessment of the pharmacists who are undertaking this service. The reasons why the service has developed in some hospitals and not others are not known. In order to extend this service, funding, resources and skillmix maximisation need to be considered. This will enable patients to gain the maximum benefit from this service development.
\end{abstract}

Department of Pharmacy and Pharmacology, University of Bath

Rachel J. Hobson, teacherpractitioner pharmacist Graham J. Sewell, professor of clinical pharmacy and pharmacy practice

Correspondence: Mrs Hobson, Department of Pharmacy and Pharmacology, University of Bath, Claverton Down, Bath, England BA2 7AY. E-mail: prxrjh@bath.ac.uk

Acknowledgments: The authors would like to thank Dr Gordon Taylor, medical statistician, for advice on statistical analysis, and the pharmacists who completed the postal questionnaire.

\section{Introduction}

The recent Audit Commission report "A spoonful of sugar" ${ }^{1}$ identified the pharmacist as a central figure in medicines management. The report stated that pharmacists should concentrate on their clinical, patient-centred roles, to help minimise medication errors and manage risk. It also advised that pharmacists should reduce their traditional role of retrospective prescription monitoring in favour of prospective, proactive roles.

This report echoes recommendations in the Review of Prescribing, Supply and Administration of Medicines report from $1999,{ }^{2}$ which advocated extending prescribing rights to pharmacists.

Development of pharmacist prescribing in the UK can benefit from the experience gained in the United States. Pharmacist prescribing was first introduced in California in the late $1970 \mathrm{~s}$. Since then, it has been extended to at least 16 states. Only one state (Florida) has introduced independent prescribing, where pharmacists are prescribing from a limited list of drugs. ${ }^{3}$ Collaborative drug therapy management has become the 
suggested model of pharmacist prescribing, whereby the pharmacist has a collaborative arrangement with a physician to dependently prescribe certain medications as agreed in a management plan. ${ }^{4-6}$

A similar model, termed supplementary prescribing, is now being implemented in England, Wales and Scotland following wide consultation. ${ }^{7,8}$ Training for pharmacists in England and Wales for supplementary prescribing will start by mid-2003. ${ }^{9}$ Supplementary prescribing will involve an agreement between independent prescribers (diagnosing) supplementary prescribers and the patient, and will be based on a clinical management plan (CMP).

Since the late 1990s many hospital pharmacy departments have strived to re-engineer their employees' roles in order to provide a better service to patients. Suggested service developments have included pharmacists transcribing discharge prescriptions, ${ }^{10-12}$ which is similar in principle to supplementary prescribing. ${ }^{13,14}$

The advantages of such schemes include increased doctor and nurse time for other activities, increased number of pharmacist interventions, increased prospective interventions, decreased prescription turnover time, cost savings (as patient's own drugs [PODs] are used more often), decreased out of hours work for the pharmacy department, and decreased error rate. ${ }^{1,13-20}$

Pharmacist transcribing of discharge prescriptions on clinical team ward rounds has been found to reduce the error rate on the prescriptions when compared with those written by doctors, to double the intervention rate by the pharmacist, and to increase the number of prospective interventions that the pharmacist makes. ${ }^{19}$ These findings fall into line with the recommendations of the Audit Commission report. ${ }^{1}$

Realisation of the benefits and the developments that can arise from such service provision could also help pharmacists gain acceptance within the clinical team as a provider of pharmaceutical knowledge, and lead to further development of the pharmacist's role.

The disadvantages of such a service relate to resource issues. For the service to be implemented throughout a hospital, it would be necessary to maximise the technician role in order to release pharmacist time from the dispensary and other duties.

One of the key elements of the British Government's White Paper "Information for Health" 21 is the implementation of Level 3 electronic prescribing within 100 per cent of acute hospitals by 2005 . Following that, there will still be a role for the pharmacist to decide upon the appropriateness of treatment on discharge and to input the discharge prescription onto the computer. Pharmacists are already using electronic prescribing systems in this manner, and have become more integrated into the healthcare team as a result. ${ }^{22-24}$

Several UK reports have identified other pharmacist prescribing roles, such as in pre-admission clinics to obtain patient medication histories and write the inpatient drug charts as well as the discharge prescription according to set protocols ${ }^{25-27}$ and also outpatient clinic prescribing. ${ }^{28}$

A survey conducted in $1999^{29}$ identified the services that hospital pharmacies were providing to facilitate seamless care upon patient discharge. The study established that out of 162 hospital trusts, one-third involved pharmacists writing discharge prescriptions, but their overall impact on the total number of prescriptions written was negligible. That survey did not attempt to suggest reasons for this, but did report that managers stated that there were continuing resource and staffing difficulties. The survey did not aim to describe the pharmacist prescribing services.

A literature review undertaken before our survey was developed did not identify any surveys that gave quantitative data on PDPTS provision in the UK.

The objectives of this survey were to identify the frequency of PDPTS provision in the UK and to provide detail of the level and type of service provided and the training requirements for pharmacists involved in such services.

\section{Methods}

\section{Questionnaire development}

Pharmacists from two hospitals with an existing PDPTS were visited to inform the development of a written, selfcompletion questionnaire containing a mixture of open and closed questions. Construction of the questionnaire was also aided by a literature review undertaken before the survey was developed. The review elicited the pharmacist "prescribing activities" that departments were then questioned about in the survey. These included PDPTS, transcribing inpatient drug charts, various prescribing activities in pre-admission clinics (which often includes transcribing discharge prescriptions $^{26,27}$ ) and prescription amendment policies.

For the purposes of this study, and in the absence of a recognised definition of transcribing, transcription is defined as "a process where a pharmacist copies a list of drugs that has been prescribed by a doctor from one chart to another chart or prescription". Although this definition implies that the pharmacist merely copies existing treatment instructions, in undertaking the act of transcription, there is an implied professional obligation on the pharmacist to review the prescribed medicines and act upon any errors and assure suitability for the patient.

The questionnaire was divided into five sections. Section A inquired about general demographic data. Section B inquired about different prescribing roles undertaken. The rest of the questionnaire sought to establish the details of PDPTS provision. Section C inquired about the extent of the service provision among those hospitals that offered a transcription service (ie, directorates/wards covered and operating hours of the service), and the model of service used. Section D covered training issues (eg, reassessment, types of training used) and Section E aimed to quantify the service provided (eg, number of prescriptions written/day/ pharmacist, advance notice required). Confidentiality was maintained by number-coding the questionnaires.

\section{Validation and piloting}

Face-to-face interviews with pharmacists (from hospitals with and without a PDPTS) were used to validate the 
questionnaire. These hospitals were chosen from the same region as the researcher for ease of travel. The questionnaire was then piloted in 20 randomly chosen hospitals from Wales, Scotland, England and Northern Ireland in July, 2001. Minor adjustments to the instructions for completing the questionnaire were made. Data collected from the pilot questionnaires were not included in the final analysis. Advice on data analysis was sought from a statistician.

\section{Main survey}

The questionnaire was distributed at the end of August, 2001, to each NHS trust in the UK providing acute hospital services. Single hospitals from each trust were identified using a combination of the UK Drug Information Pharmacists' Group Directory and the Chemist \& Druggist Directory. ${ }^{30}$ The questionnaire was sent to one hospital from each trust. Any hospitals that were found to have merged with another trust were removed from the database.

The questionnaire was accompanied by a letter which included recognised descriptions of independent and dependent (now supplementary) prescribing, ${ }^{2}$ and the researcher's definition of transcribing, in order to clarify recipient's understanding of the different types of prescribing.

The covering letter was addressed to the chief pharmacist, principal pharmacist or clinical services manager. The recipient was requested to pass the questionnaire on to the most relevant person to complete (if it was not themselves). A freepost-addressed envelope was included for return of the questionnaire.

No deadline for completion of the questionnaire was stated on the questionnaire or covering letter, but nonrespondents were followed up by a telephone call after three weeks and then again at six weeks. Further copies of the questionnaire were sent out to those who requested them. The final deadline for accepting returned questionnaires was 11 weeks after they had been originally posted.

Data obtained from returned questionnaires were coded and analysed using the Statistical Package for the Social Sciences (SPSS) version 10, and the significance of the association between variables was assessed using chi-squared, Kruskal-Wallis and bivariate correlations (Spearman's rho), where appropriate.

\section{Results}

\section{General demographics}

A total of 234 hospital pharmacy departments were identified, of which 20 were used for piloting the questionnaire, leaving 214 hospitals for the main study. Eight of these hospitals were removed after it was established that they had merged with another Trust, leaving 206 hospitals eligible for the study. Of these 206 hospitals, responses were received from 135 (66 per cent response rate). Sixtyeight per cent of responses $(n=92)$ came from district general hospitals, 27 per cent $(\mathrm{n}=37)$ from teaching hospitals, and 4 per cent $(n=5)$ from tertiary referral centres.

The questionnaire was completed by clinical pharmacist/managers ( 26 per cent, $n=35$ ), chief pharmacists ( 26 per cent, $\mathrm{n}=35)$, principal pharmacists $(25$ per cent, $\mathrm{n}=34$ ), pharmacy managers ( 7 per cent, $\mathrm{n}=10$ ), deputy chief pharmacists ( 5 per cent, $\mathrm{n}=7$ ), medicines information manager/pharmacists ( 4 per cent, $n=5$ ), discharge services pharmacist ( 4 per cent, $\mathrm{n}=5$ ) and one interface pharmacist (1 per cent).

The size of the hospitals varied, with bed sizes ranging from $<100$ to $>1,500$, with the most common range being 401-600 (33 per cent, $n=44$ ).

\section{Prescribing activities}

\section{PDPTS}

Thirty-six per cent of departments $(49 / 135)$ were currently offering a pharmacist discharge prescription transcription service. This was the most common prescribing activity undertaken by pharmacists. Twenty departments ( 15 per cent) reported that they transcribed inpatient drug charts. The majority of these departments $(17 / 20,85$ per cent) also transcribed discharge prescriptions. No tertiary referral centres transcribed.

\section{Prescription amendment policy}

The second most common pharmacist prescribing activity was a prescription amendment policy $(39 / 135,29$ per cent) whereby the pharmacists could change timings and frequencies of drugs or change a non-formulary drug to a formulary alternative within the same pharmacological class, following agreed protocols.

\section{Pre-admission clinics}

Twenty-four of the 135 departments (18 per cent) reported that they performed prescribing roles in pre-admission clinics. Twenty departments stated that pharmacists wrote patients' normal medication onto drug charts. Twelve departments reported that pharmacists prescribed medicines on to a drug chart at pre-admission clinics according to set protocols, including analgesia, antibiotics and venous thromboembolism prophylaxis, and eight departments prescribed discharge medication at preadmission clinics according to set protocols. Six departments performed two of these roles and five departments performed three of these roles.

\section{No prescribing activity}

Hospitals with no pharmacist prescribing comprised the largest group of respondents $(n=59,44$ per cent).

\section{Other prescribing}

The most common "other" form of pharmacist prescribing that was reported was prescribing in anticoagulant clinics (10 per cent, $n=13$ ) and total parenteral nutrition (TPN) prescribing ( 3 per cent, $n=4)$. Four departments reported that they had pharmacists that independently prescribed. Other pharmacist prescribing included chemotherapy, in cardiac rehabilitation clinics, migraine 
clinics, any "P" medicines and medicines that the patient had been taking before admission.

\section{Future plans}

Of the 86 departments not offering a PDPTS, 36 (42 per cent) indicated that there had been discussions about pharmacist transcribing, but no decision had been made as yet. Thirty-four per cent $(n=29)$ indicated that there were no plans for such a development, and 22 per cent $(n=19)$ said that they were currently developing such a service $(2=$ missing data $)$.

Eleven of the departments that said they were implementing a transcription service intended to implement the service in 2002, and two departments intended to implement the service in 2003. One department intended to implement the service during December 2001 (5=missing data).

Of the 86 non-transcribing pharmacy departments, 59 (69 per cent) undertook no prescribing activity (range $=0$ to 3 prescribing activities). Transcribing departments offered a wider range of prescribing activities (range $=1$ to 8 prescribing activities).

A weak relationship was found between the total number of pharmacists employed per hospital and the total number of prescribing activities undertaken (correlation coefficient $=0.208, P=0.018$ ).

\section{Prescribing systems}

Asked when the pharmacy departments started their transcription service, one hospital stated that they had been running such a service since the 1980s; all of the other hospitals with a PDPTS had started the service between 1995 and 2001.

The majority (68 per cent, 32/47) operated the service during normal working hours, Monday to Friday. A few hospitals had extended to parts of the weekend or later in the evenings, but this was an exception.

The wards/directorates in which the PDPTS was offered are illustrated in Figure 1. The most common directorate to have a PDPTS was the medical directorate, with 51 per cent of hospitals (24/47) running the service

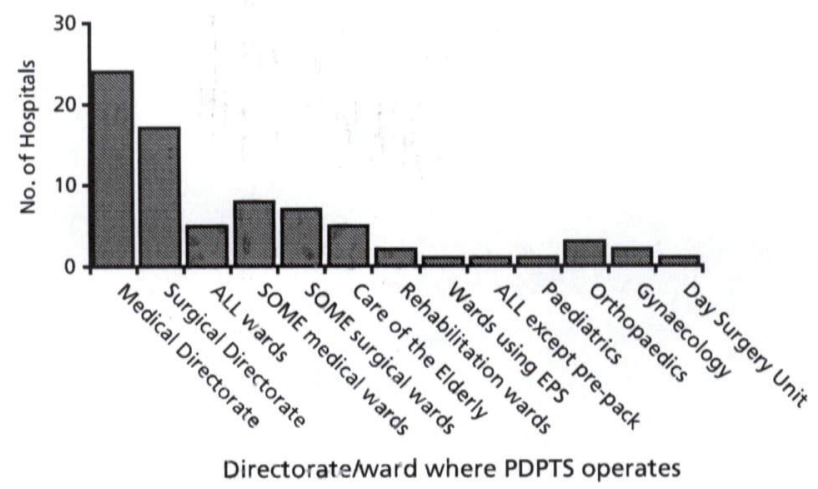

Figure 1 Directorates/wards where PDPTS operates $(n=47)$. within this directorate. The next most common directorate was the surgical directorate ( 36 per cent, 17/47). Only 11 per cent of hospitals $(5 / 47)$ had rolled out the service to all wards, and one hospital provided the service to all wards minus those wards that stocked pre-packed drugs. One hospital operated PDPTS only in those wards where an electronic prescribing system (EPS) was in place.

The pharmacy departments mainly funded the PDPTS (58 per cent, 28/48). Some services were funded by the medical and/or surgical directorates (23 per cent, 11/48) and some had received trust monies into the pharmacy budget ( 8 per cent, $4 / 48$ ).

The number of pharmacists providing PDPTS per hospital ranged from 1 to $89 \quad($ mean $=8$, median $=5$, mode $=2,25 \%$ percentile $=2,75 \%$ percentile $=10$ ). The total number of pharmacists (whole time equivalent) per department that provided PDPTS ranged from 7 to 102 $($ mean $=19$, median $=16$, mode $=9)$. The percentage of pharmacists involved in PDPTS per department ranged from 3 per cent to 100 per cent $($ mean $=39$ per cent, median $=33$ per cent, mode $=33$ per cent).

The models of PDPTS in use are shown in Table 1. The first four models of service were established from site visits and the other categories were identified from the results of the "other" option in the questionnaire, from which common themes were identified. The most common model used was the ward pharmacist model ( 78 per cent) in which pharmacists transcribed the discharge prescriptions for their own ward.

The majority of departments (79 per cent, 37/47) reported using paper-based prescriptions for PDPTS. Pharmacists produced prescriptions on electronic prescribing systems in nine departments (six used computergenerated prescriptions and three used both paper and computer systems).

Table 2 illustrates the number of discharge prescriptions pharmacists reported transcribing per day. The

Table 1 Models of PDPTS in use $(n=49)$.

\begin{tabular}{ll}
\hline Model & Frequency (\%) \\
\hline $\begin{array}{l}\text { Pharmacists attend whichever ward } \\
\text { bleeps them }\end{array}$ & $3(6)$ \\
Ward pharmacist model & $38(78)$ \\
Medical pharmacist attends whichever & $8(16)$ \\
ward within the medical directorate & \\
bleeps them & $4(8)$ \\
$\begin{array}{l}\text { Surgical pharmacist attends whichever } \\
\text { ward within the surgical directorate } \\
\text { bleeps them }\end{array}$ & $1(2)$ \\
Ward model plus urgent bleeps \\
$\begin{array}{l}\text { Ward model plus only at specific } \\
\text { times/ward round }\end{array}$ \\
$\begin{array}{l}\text { Ward model plus odd prescriptions } \\
\text { written in pharmacy }\end{array}$ \\
$\begin{array}{l}\text { The total is greater than } 49 \text { as some respondents ticked more than } \\
\text { one option for this question. }\end{array}$
\end{tabular}


Table 2 Number of pharmacist-written prescriptions/pharmacist/ day $(n=48)$.

\begin{tabular}{ll}
\hline Number of prescriptions/day & Frequency (\%) \\
\hline$<5$ & $25(52)$ \\
$5-10$ & $17(35)$ \\
$11-15$ & $3(6)$ \\
$16-20$ & $2(4)$ \\
$26-30$ & $1(2)$ \\
Total & $48(100)$ \\
\hline
\end{tabular}

majority of pharmacists ( 52 per cent) said they were writing less than five prescriptions per day and 35 per cent were writing 5-10 prescriptions per day.

The advance notice required by the pharmacy department to produce a pharmacist-written discharge prescription is shown in Table 3.

\section{Training}

Training requirements for pharmacists who transcribe discharged prescriptions were explored (Table 4). The most common training requirement was the completion of an in-house training programme ( 55 per cent), followed by designation by a senior pharmacist (31 per cent), and possession of a clinical diploma ( 20 per cent). Fourteen per cent of hospitals reported no additional training requirements.

Twenty-seven hospital pharmacy departments reported that they had an in-house training programme for PDPTS, but only eight of these reported having a formal training programme. Of these, five used tutorials, seven used observation, seven used supervision, and four used an examination (some departments used a combination of techniques).

Of the 11 departments that said that they assessed competency to transcribe, four did this via non-ward based training/assessment, four departments carried out ward-based assessment and one completed an annual competency review $(2=$ missing data $)$.

Reported frequency of re-assessment of competency of the pharmacists who were transcribing is shown in Table 5. One-third of departments $(n=15)$ did not conduct re-

Table 3 Advance notice required to produce a prescription $(\mathrm{n}=48)$

\begin{tabular}{ll}
\hline Advance notice required & Frequency (\%) \\
\hline Less than 1 hour & $16(33)$ \\
1-2 hours & $6(13)$ \\
$3-4$ hours & $1(2)$ \\
24 hours & $6(13)$ \\
No rule as such & $13(27)$ \\
Only written while pharmacist is on the ward & $6(13)$ \\
Total & $48(100)$ \\
\hline
\end{tabular}

Table 4 Training required for pharmacists to transcribe $(n=49)$.

\begin{tabular}{ll}
\hline Training & Frequency (\%) \\
\hline Clinical certificate & $8(16)$ \\
Clinical diploma & $10(20)$ \\
MSc in clinical pharmacy & $1(2)$ \\
Designation by senior pharmacist & $15(31)$ \\
2 years' ward experience & $7(14)$ \\
3 years' ward experience & $1(2)$ \\
In-house training programme & $27(55)$ \\
No further training & $7(14)$ \\
At least 1 year of diploma completed & $1(2)$ \\
Clinical diploma or 3 years' experience & $1(2)$ \\
Training programme in development & $1(2)$ \\
\hline
\end{tabular}

The total is greater than 49 as some respondents ticked more than one option for this question.

Table 5 Frequency of reassessment of pharmacists providing a PDPTS $(n=46)$.

\begin{tabular}{ll}
\hline Frequency of reassessment & Frequency (\%) \\
\hline Twice a year & $2(4)$ \\
Once a year & $1(2)$ \\
Once every 2 years & $3(7)$ \\
Never reassess & $15(33)$ \\
Not reached a decision & $22(48)$ \\
On-going assessments & $3(7)$ \\
Total & $46(100)$ \\
\hline
\end{tabular}

assessment. The nine departments that did undertake some form of re-assessment were asked how they did this. Three departments used observation and two used a "total competency assessment programme". One department used supervised transcription of discharge prescriptions, one used an examination, one used on-going assessment via an intervention programme, and one completed an audit of prescriptions prepared by the pharmacist.

\section{Discussion}

\section{Critique of method}

The response rate was slightly lower than similar questionnaire surveys ${ }^{29,31}$ but this may be due to the fact that the questionnaire was sent out in the summer. It is possible that sending the questionnaire out at this time of year could have introduced an element of bias but this seems unlikely as the data collection period was 11 weeks and so staffing bias was not expected.

Difficulties obtaining an up to date list of clinical pharmacists resulted in some questionnaires being directed to chief pharmacists and principal pharmacists. This may have affected the response rate and also the information in the response, and so may have led to some bias. A 
manager, such as a chief pharmacist or a principal pharmacist, would be indirectly involved with the service whereas a clinical pharmacist would be directly involved with the dayto-day running of a transcription service. Another problem was identifying hospitals where trusts had merged. Some of these were not identified until questionnaires were returned; these hospitals were then removed from the results.

It would have been preferable to use a sampling method whereby the questionnaire was sent to every hospital in every trust rather than one of them. This is because some trusts have only recently merged and may have different pharmacist prescribing roles and transcription services in place from the other hospital(s) in the trust. However, time constraints meant that the number of questionnaires would have been too great to deal with.

There are several areas where it has become apparent that further questioning would have been useful:

- The percentage of the overall prescriptions written in the hospital that were written by pharmacists

- Is any other type of pharmacist assessment undertaken? Some hospitals commented that although they did not complete an assessment specifically for pharmacists' transcription abilities, they did complete a whole competence assessment regularly

- Is the PDPTS regularly audited?

- Opinions about the impact of electronic prescribing on PDPTS

- Reasons for lack of further extension of PDPTS.

\section{Prescribing activities}

Just over half the departments had undertaken some form of pharmacist prescribing. The most common type of prescribing was transcribing discharge prescriptions, followed by prescription amendment, pre-admission clinics and rewriting drug charts. Hospitals that offered a PDPTS also offered a wider range of other pharmacist prescribing activities than those hospitals not offering such a service.

\section{Prescribing systems}

The ward model of pharmacist transcribing was the most commonly used (78 per cent). Ideally, the ward pharmacist should be writing discharge prescriptions while on the ward round, when medicine management issues can be discussed with the whole team as a collaborative process. $^{4,6}$ The pharmacist could write the discharge prescriptions as the ward round is continuing, meaning that as soon as the discharge decision is made, the prescription can be written and passed on to ward technicians to process.

The majority of pharmacists (52 per cent) who were transcribing discharge prescriptions were writing less than five prescriptions per day. As over half of the hospitals had less than five pharmacists who transcribed prescriptions, it can be assumed that the majority of hospitals who ran such a service were not having a large impact on the overall number of discharge prescriptions being written in the hospital. This agrees with the findings of Sexton's UK survey of 1999, which found that pharmacists were involved in writing discharge prescriptions in about onethird of hospitals, but their impact was considered to be negligible on prescribing overall. ${ }^{29}$

The transcription services were mainly operating during normal working hours Monday to Friday. Slee and Farrar $^{32}$ showed that on weekdays 50 per cent of inpatient and 18 per cent of take-home prescriptions were written outside the traditional $9 \mathrm{am}-5 \mathrm{pm}$ working day. Thus the transcribing service may not be meeting patient needs. If a transcription service is to have a significant impact, it needs to be operated over extended hours. The optimum benefit from this service provision may actually be from $5 \mathrm{pm}$ until midnight when junior doctors are working with minimal senior support. It could be hypothesised that this time would pose the maximum risk for prescribing errors.

\section{Training}

Even though all pharmacists who are competent to practice should be able to transcribe discharge prescriptions, the requirements of clinical governance mean that the service should be quality assured and accountable. ${ }^{33}$ All pharmacists providing the service should be assessed against key competencies, to provide a consistent service of a suitable standard.

A relatively low number of hospitals $(n=8)$ had a specific and formalised training programme. This finding suggests that when 55 per cent of respondents said that they asked their pharmacists to undertake an in-house training programme in order to be authorised to transcribe, some of these "programmes" may be ad-hoc arrangements.

The service should also be regularly audited to make sure that standards are being maintained. Principles of clinical governance are not being adhered to if these issues are not addressed.

\section{Future research}

Further research is needed to investigate the different models of pharmacist prescribing and the impact of this service development upon the pharmacy department, to enable hospitals that are not currently offering pharmacist prescribing services to introduce the optimum service model. Also, an economic study to investigate the cost/ benefit ratio of providing such a service should be undertaken.

\section{Conclusion}

A pharmacist discharge prescription transcription service is being operated in around one-third of UK hospitals. The transcription services offered tend to be rather ad-hoc and only available in certain wards or single directorates. The reasons for this limited availability are unknown. Training and competency assessment of this role appear not to meet the requirements of clinical governance. 
Published literature supports the patient benefits of such a service in terms of reduced waiting time for medicines on discharge ${ }^{14,16,18,20}$ and also reduced error rates. ${ }^{1,9,14,19}$ In order to extend this service, funding, resources and skillmix maximisation need to be considered. This will enable patients to gain the maximum benefit from this service development.

\section{References}

1 Audit Commission. A spoonful of sugar - Medicines management in NHS hospitals. London: Audit Commission; 2001.

2 Review of prescribing, supply and administration of medicines, final report. London: Department of Health; 1999.

3 Farrell J, North-Lewis P, Cross M. Pharmacist prescribing in the United States. Pharm J 1997;259:187-90.

4 Carmichael JM, O'Connell MB, Devine B, Kelly W, Ereshefsky L, Linn WD, et al. Collaborative drug therapy management by pharmacists. Pharmacotherapy 1997; 17:1050-61.

5 Webb CE. Prescribing medications: Changing the paradigm for a changing health care system. Am J Health Syst Pharm 1995;52:1693-5

6 Galt KA. The key to pharmacist prescribing: Collaboration. Am J Health Syst Pharm 1995;52:1696-9.

7 Proposals for supplementary prescribing by nurses and pharmacists and proposed amendments to the Prescription Only Medicines (Human Use) Order 1997 (MLX 284). London: Department of Health/Medicines Control Agency; 2002.

8 The Right Medicine: A strategy for pharmaceutical care in Scotland. Edinburgh: Scottish Executive; 2002:1-38.

9 Root G. Supplementary prescribing - a groundbreaking opportunity. Pharm J 2003;270:19-20.

10 Taheny K, Tyrell A, Cairns C, Bunn R. Influence of patient focused care on managing medicines for discharge. Pharm $\mathrm{J}$ 1999;262:368-71.

11 Leech D. Patient directed pharmacy services - do they benefit their patients? Hosp Pharm 2000;7:263-4.

12 Leech D. New roles for pharmacy technicians at the William Harvey Hospital. Hosp Pharm 2000; 7:30-2.

13 Ketley D, Qualie MJ, Godfrey BD. The near patient clinical pharmacist 1. From design to pilot. Pharm J 1998;261:247-9.

14 Jacklin A, Patel K, Almossawi O. A discharge pharmacist service improves the timeliness, quality and cost of discharge. Pharm Pract 2001;11(April):100-2.
15 Qualie MJ, Godfrey BD, Ketley D. The near patient clinical pharmacist 2. From pilot to service. Pharm J 1998;261:287-9.

16 Culshaw M, Dawes S. Assessing the value of a discharge pharmacist. Pharm Manage 1998;14:22-3.

17 Feil RA, Jones WN. The impact of pharmacist-initiated discharge prescription writing on adverse drug reactions and medical centre drug costs. Pharmacotherapy 1999;19:509.

18 Milliken B, Rea Y. A clinical pharmacist's contribution to discharge planning and patient counselling. Pharm J 1997;258:777-8.

19 Boorman S, Cairns C. Another way forward for pharmaceutical care: a team-based clinical pharmacy service. Pharm J 2000;264:343-6.

20 Cattell R, Conroy C, Sheikh A. Pharmacist intergration into the discharge process: a qualitative and quantitative impact assessment. Int J Pharm Pract 2001;9:59-64.

21 NHS Executive. Information for health: an information strategy for the modern NHS 1998-2005. London: Department of Health; 1998.

22 Williams C. Electronic prescribing can increase efficiency of the discharge process. Hosp Pharm J 2000;7:206.

23 Farrar K. Can electronic prescribing increase the efficiency of the discharge process? Hosp Pharm 2000;7:114.

24 Norris C, Thomas V, Calvert P. An audit to evaluate the acceptability of a pharmacist electronically prescribing discharge medication and providing information to GPs. Pharm J 2001;267:857-9.

25 Lord $\mathrm{S}$. The interface - Steps towards seamless care. Hosp Pharm 1999;6:83-4.

26 Hebron B, Jay C. Pharmaceutical care for patients undergoing elective ENT surgery. Pharm J 1998;260:65-6.

27 Hick H, Deady PE, Wright D, Silcock J. The impact of the pharmacist on an elective general surgery pre-admission clinic. Pharm World Sci 2001;23:65-9.

28 Booth CD. Pharmacist-managed anticoagulant clinics: A review. Pharm J 1998;261:623-5.

29 Sexton J, Ho YJ, Green CF, Caldwell NA. Ensuring seamless care at hospital discharge: a national survey. J Clin Pharm Ther 2000;25:385-93.

30 Grice P, editor. Chemist \& Druggist Directory: London: Miller Freeman; 1998.

31 Wilkie P, Sibbald B, Raftery J, Anderson S. Prescribing at the hospital-general practice interface I: Hospital outpatient dispensing policies in England. BMJ 1992;304:29-31.

32 Slee A, Farrar K. What are the normal working hours for a hospital pharmacy? Pharm J 1998;260:923-5.

33 Achieving excellence in pharmacy through clinical governance. London: Royal Pharmaceutical Society of Great Britain; 1999. 